\title{
Drug Utilization Review of Cholinesterase Inhibitors in Quebec
}

\author{
Fadi Massoud, Marc Dorais, Claudie Charbonneau, Bénédicte Lescrauwaet, \\ Jean-Marc Boucher, Jacques LeLorier
}

Can. J. Neurol. Sci. 2008; 35: 508-509

Dementia affects $9 \%$ of the population over the age of $65 .^{1}$ Most cases of dementia (65\%) are due to Alzheimer's disease (AD). Three cholinesterase inhibitors (ChEI) are currently available on the Canadian market for the treatment of $\mathrm{AD}$ : donepezil, rivastigmine, and galantamine. Very few environmental studies exist on the use of these medications in a nonselected population of patients with $\mathrm{AD}$, and research on the determinants of persistence and compliance with ChEI's in patients suffering from AD has not been pursued extensively. We propose to review the utilization of ChEI's in all individuals who qualified for reimbursment by the Régie de l'assurance maladie du Québec, the government body responsible for medical insurance in the province of Quebec.

\section{Material AND Methods}

In Quebec, ChEI's are reimbursed according to specific clinical criteria as "exception medications" based on a diagnosis of mild to moderate AD (Folstein's Mini-Mental Status Examination between 10-26, and global evaluations of cognition, function, behaviour, and social interactions). The objectives of our study were to examine the characteristics of patients being prescribed ChEI and the variables associated with continued therapeutic use (or not). The data were extracted from the administrative databases of Régie de l'assurance maladie du Québec which contain information on demographic variables, medical services, and all prescribed drugs dispensed to the elderly (65 years or older), social security beneficiaries, and, since January 1997, all Quebec residents not covered by a private drug insurance plan. This database included $91.1 \%$ of individuals 65 years and older in 2003. However, it gives no information on measures of cognition, functional status, nor the reasons for discontinuation of therapy. The recruitment period includes all subjects receiving at least one dispensation of ChEI between April 1st, 2000 and June 30th, 2003. These dates are based on the timing of reimbursement of ChEI's in Quebec. The index date corresponds to the first dispensation of ChEI during the recruitment period. We evaluated descriptive statistics of characteristics of new users at the index date. Discontinuation of therapy was defined as non renewal of any ChEI before the end of the grace period ( $50 \%$ of the prescription duration) that was added to the end of the prescription. Kaplan-Meier analysis was used to analyze persistence rates and a Cox proportional hazard model was employed to estimate the risk factors of discontinuation. This analysis was adjusted for age, gender, speciality of the physician initiating the therapy, number of drug
Table: Adjusted rate ratios for therapy discontinuation
Variable

Index Drug

Galantamine

Rivastigmine

Donepezil

Age

40 to 69 years

70 to 79 years

80 to 89 years

90 years and over

Gender

Female

Male

Chronic Disease Score

Resource use/person in the year prior to the index date

Number of hospitalizations (all causes)

Number of days of hospitalization

General practitioner visits

Specialist visits (all specialists)

Emergency room visits

Status of physician prescribing the index drug

Specialist

General practitioner
Rate Ratio (95\% CI)

1.00 (reference)

$1.09(0.97-1.22)$

$1.13(1.04-1.24)$

1.00 (reference)

$1.14(0.99-1.30)$

$1.23(1.08-1.40)$

$1.44(1.21-1.71)$

1.00 (reference)

$1.03(0.97-1.10)$

$0.99(0.98-0.99)$

$0.98(0.96-1.00)$

$0.99(0.98-0.995)$

$0.99(0.99-1.01)$

$1.01(1.00-1.01)$

$1.01(0.99-1.03)$

1.00 (reference)

$1.09(1.01-1.18)$
From the Service de Gériatrie (FM, JMB), Pharmacoeconomic and

Pharmacoepidemiology Research Unit (MD, JL), Centre de Recherche du Centre Hospitalier de l'Université de Montréal, Département de Médecine (FM, JMB, JL), Centre Hospitalier de l'Université de Montréal (CHUM), Montreal, QC; Pfizer Inc. (CC), New York, NY, USA; Formerly of Pfizer Canada Inc. (BL), Kirkland, Quebec. Received February 20, 2008. Final Revisions Submitted April 23, 2008. Correspondence to: Fadi Massoud, Hôpital Notre-Dame, Service de Gériatrie, 1560 rue Sherbrooke est, Montréal, Québec, H2L 4M1, Canada. 
dispensations in the year prior to the index date, health care services use per person in the year preceding the index date and the Chronic Disease Score (CDS) (this score is derived from drugs dispensed to the patient depending on the severity of the disease for which they have been prescribed). ${ }^{2}$ This score was updated by our group for the purpose of this study.

\section{Results}

At least one dispensation of ChEI was received by 18,748 individuals and the index drug was donepezil for $81 \%$, rivastigmine for $11 \%$, and galantamine for $8 \%$. The mean age was 79.2 years and $68 \%$ were women. In the year prior to the index date: the mean CDS was 3.6, the mean number of total drug dispensations was 64 , the mean number of hospitalizations was 0.8 , the mean number of days of hospitalizations was 2.5, the mean number of visits was 5.6 to a general practitioner (GP) and 4.2 to a specialists. At the index date, $74 \%$ of the prescriptions were made by a GP, $15 \%$ by a neurologist, $5 \%$ by a geriatrician, and $1 \%$ by a psychiatrist. Fifty percent of patients were still taking their index medication 216 days after initiation. One year after the initiation of a ChEI, $40 \%$ of patients were still on therapy. The risk of discontinuation (Table) was significantly higher for patients initiated with donepezil compared to galantamine (rate ratio $=1.13$ ), for patients aged 80 years or more compared to those aged less than 70 years, and for patients who received their index drug from a GP (rate ratio=1.09).

\section{Discussion}

Most individuals being prescribed ChEI's received donepezil. They are in general good health and use relatively few medical resources. These medications are well tolerated and $50 \%$ of patients are still taking their index medication 216 days after initiation. Few studies have evaluated use and persistency of ChEI in a "real-world" setting. Three studies relatively similar to ours in design were identified. ${ }^{3-5}$ Theses studies are not readily comparable considering study populations, health-care system differences, and durations of follow-up. However, certain observations can be made. Similarly to our study, donepezil was the most commonly used index drug in all other studies. This can probably be explained by the fact that donepezil was the first of the three drugs to be marketed on the North-American market. When compared to a study in Ontario, ${ }^{3}$ individuals in the Quebec cohort were in better general health as suggested by fewer medical visits and drug prescriptions. These differences could be explained, at least in part, by the fact that our study did not include individuals in long-term care who are considerably frailer. This might also explain the longer duration of use in the Ontario study in which $31.8 \%$ of individuals who died in longterm care were still using a ChEI. Another likely explanation for the longer duration of use observed in Ontario is the fact that the authors used a much longer grace period. A majority of prescriptions had a duration of 30 days and the grace period of 120 days used in the Ontario study corresponds to a grace period of $400 \%$, compared to $50 \%$ in our study. Concerning the persistency results, the two American studies only compared donepezil and rivastigmine users and did not find any difference between the two drugs. ${ }^{4,5}$ The Ontario study only presents persistency results for the whole class of drugs. In our study, factors associated with discontinuation might be related to higher expectations as patients get older (versus lower threshold for discontinuation), possible "lower exposure" to the health system reflected by a lower CDS, and initiation by a GP who could be less familiar with the medication. In one study, ${ }^{4}$ persistency was associated with frequency of physician visits or hospitalization after initiation of treatment. It remains unclear from our study why galantamine was associated with longer persistence to treatment.

\section{ACKNOWLEDGEMENTS}

Dr. Jean-Marc Boucher is now deceased.

This work was supported by an unrestricted grant from Pfizer Canada. Pfizer was not involved in the analyses performed nor their interpretation. They were aware of the results, but did not participate in writing nor approval of this manuscript.

\section{CONFliCt OF InTEREST}

Fadi Massoud has received speaker's honoraria, consultant fees, and research support from Pfizer, Janssen Ortho Inc., and Novartis. Miss Claudie Charbonneau is presently employed by Pfizer US and Miss Bénédicte Lescrauwaet was employed by Pfizer at the time of the study.

\section{REFERENCES}

1. Canadian study of health and aging. Study methods and prevalence of dementia. Can Med Assoc J. 1994;150:899-13.

2. Von Korff M, Wagner EH, Saunders K. A chronic disease score from automated pharmacy data. J Clin Epidemiol. 1992;45: 197-203.

3. Herrmann N, Gill SS, Bell CM, Anderson GM, Bronskill SE, Shulman KE, et al. A population-based study of cholinesterase inhibitor use for dementia. J Am Geriatr Soci. 2007;55:1517-23.

4. Suh DC, Thomas SK, Valiyeva E, Arcona S, Vo L. Drug persistency of two cholinesterase inhibitors: rivastigmine versus donepezil in elderly patients with Alzheimer's disease. Drugs Aging. 2005; 22:695-07.

5. Singh G, Thomas SK, Arcona S, Lingala V, Mithal A. Treatment persistency with rivastigmine and donepezil in a large state medicaid program. J Am Ger Soc. 2005;53:1269-70. 